\title{
A Rare Case of Biliary Peritonitis After Colonoscopy Without Any Perforation of The Biliary Tract
}

\author{
Yoshihito Souma*, Takeyoshi Yumiba, Jun Yasuda, Junji Ieda, Susumu Miyazaki, Tomojiro Ono, \\ Tetsuro Kobayashi, Toru Saito and Riichiro Nezu
}

Department of Surgery, Osaka Central Hospital, Osaka, Japan

*Corresponding author: Yoshihito Souma, Department of Surgery, Osaka Central Hospital 3-3-30 Umeda, Kita-ku, Osaka 530-0001, Japan

\section{ARTICLE INFO}

Received: 幽 December 27, 2020

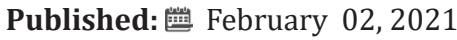

Citation: Yoshihito Souma, Takeyoshi Yumiba, Jun Yasuda, Junji Ieda, Susumu Miyazaki, Tomojiro Ono, Tetsuro Kobayashi , Toru Saito, Riichiro Nezu. A Rare Case of Biliary Peritonitis After Colonoscopy Without Any Perforation of The Biliary Tract. Biomed J Sci \& Tech Res 33(4)-2021. BJSTR. MS.ID.005423.

Keywords: Biliary Peritonitis; Bile Transudation; Colonoscopy

\begin{abstract}
Biliary peritonitis without any perforation of the biliary tract is extremely rare. An 82-year-old female was admitted to our hospital with severe abdominal pain one day after screening colonoscopy. Computed tomography revealed abnormal ascites dominantly in the left paracolic gutter and a swollen gallbladder with dilated common bile duct. We suspected pan-peritonitis due to the colonic perforation after colonoscopy and performed an emergency operation. On exploration, any fecal leakage, colonic perforation, nor gastrointestinal perforation were not found, but biliary ascites without any perforation of the biliary tract was found. We considered that the cause of a disease might be the possibility of bile transudation through the biliary tract, therefore, we performed cholecystectomy followed by irrigation and drainage of the abdominal cavity. Postoperative gastroduodenoscopy did not revealed any suspicious finding of perforation in the gastroduodenal tract but revealed a large parapapillary diverticulum, and postoperative magnetic resonance cholangiopancreatography did not show anomalous arrangement of pancreaticobiliary ductal system and choledocholithiasis. Postoperative course was uneventful, and she was discharged 72 days after the operation. There were thinning parts in the wall of the resected gallbladder, and bile leakage from where was not identified macroscopically. We speculated that the underlying pathology associated with increased intraductal pressure of biliary tract due to colonoscopy and long-term administration of corticosteroid might lead to the transudation of bile juice.
\end{abstract}

\section{Introduction}

Biliary peritonitis is a rare cause of acute abdomen with life threatening consequences, ant it is usually associated with the perforation of biliary tract or upper gastrointestinal tract $[1,2]$. However, there have been some reports of biliary peritonitis occurring without any detectable perforation of biliary tract [35]. Rarity of this condition often makes its diagnosis difficult, and the delay of surgical intervention will lead to increased morbidity and mortality [6-9]. Therefore, immediate diagnosis and surgical intervention are fundamental to improve the outcome of treatment for biliary peritonitis [10]. We herein describe a rare case of biliary peritonitis due to bile transudation without any perforation of the biliary tract occurred after screening colonoscopy.

\section{Case Report}

An 82-year-old Japanese female, who had a medical history of pure red cell aplasia treated with long-term prednisolone $5 \mathrm{mg} /$ day, was admitted to our hospital with complaining of severe abdominal pain one day after screening colonoscopy. On an examination, she was visibly jaundiced, tachycardic, and febrile with a temperature of $38.7^{\circ} \mathrm{C}$. The abdominal pain was continuous, and the diffuse rebound tenderness were noted without localization. Laboratory values showed the increasing of white blood cells of $15.3 \times 10^{3} / \mu \mathrm{l}$, serum C-reactive protein of $25.2 \mathrm{mg} / \mathrm{dl}$, total bilirubin of $3.9 \mathrm{mg} /$ $\mathrm{dl}$, direct bilirubin of $2.5 \mathrm{mg} / \mathrm{dl}$, aspartate aminotransferase of 220 $\mathrm{IU} / \mathrm{l}$, alanine aminotransferase of $88 \mathrm{U} / \mathrm{l}$, alkaline phosphatase of 1227 IU/l, gamma glutamyl transferase of 231 IU/l, amylase of 494 $\mathrm{U} / \mathrm{l}$, and lactate dehydrogenase of $363 \mathrm{IU} / \mathrm{l}$. Computed tomography (CT) revealed fluid collection dominantly in the left paracolic gutter, but free gas was not found within the peritoneal cavity (Figure 1A). CT also showed swollen gallbladder and dilated common bile duct (Figures 1B \& 1C). With a diagnosis of pan-peritonitis due to colonic perforation after colonoscopy, she underwent emergency 
laparoscopy to identify the causative pathology. Laparoscopic examination showed bilious ascites in the abdominal cavity concomitant with the diffuse fibro-purulent exudates around the gallbladder bed and hepatic hilum (Figure 2A), but an apparent colonic perforation and fecal leakage were not observed.

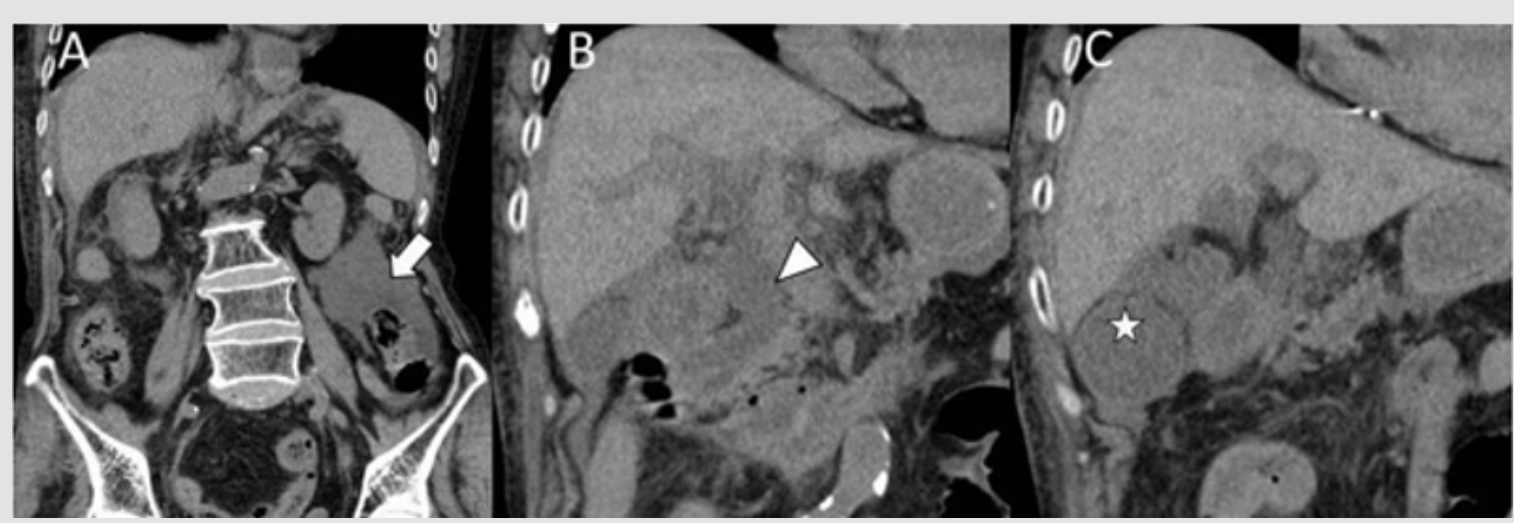

Figure 1:

A. Computed tomography (CT) revealed fluid collection in the left paracolic gutter (arrow).

B. B \& C. CT also showed swollen gallbladder (arrowhead) with dilated common bile duct (asterisk).

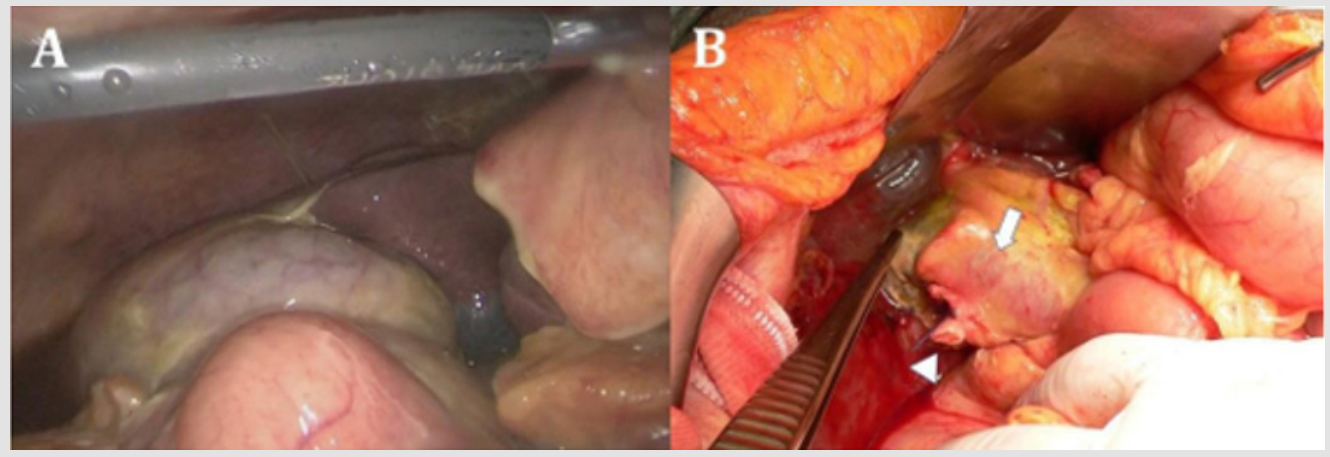

Figure 2:

A) Bilious ascites in the perihepatic space concomitant with the diffuse fibro-purulent exudates around the gallbladder bed.

B) we found bile retention around the hepatic hilum despite no obvious perforation site in the biliary tract and upper gastrointestinal tract (arrow indicates the common bile duct and arrowhead indicates stump of the cystic duct).

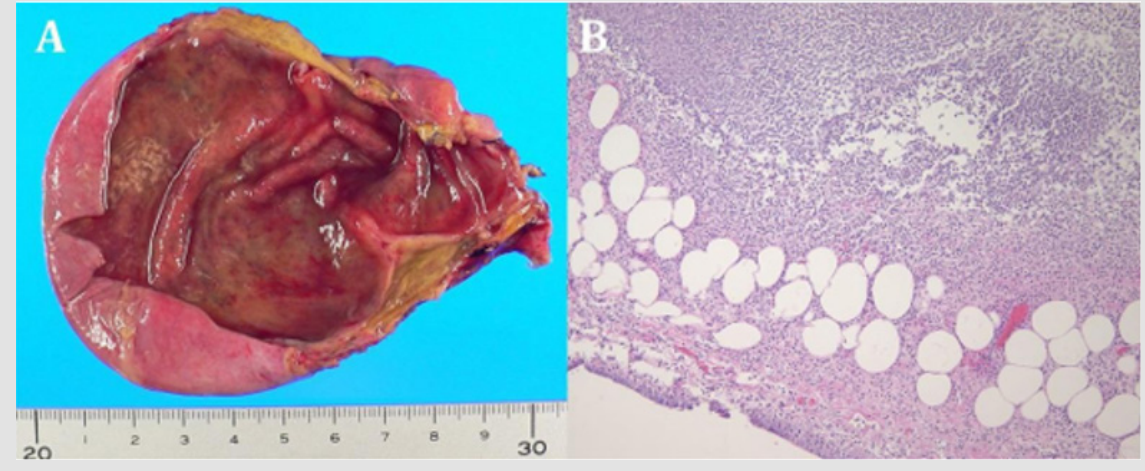

Figure 3:

A) The resected gallbladder was $9.8 \mathrm{~cm}$ long without gallstone. There were focal thinning parts in the corpus of the resected gallbladder, and bile leakage from where was not identified.

B) Histopathological findings showed an infiltration of the inflammatory cells into the wall of gallbladder. 
According to the laparoscopic findings, we diagnosed biliary peritonitis due to the perforation of the biliary tract and then converted to open surgery via the upper middle incision. On the exploration, we found bile retention around the hepatic hilum despite no obvious perforation site in the biliary tract and upper gastrointestinal tract (Figure 2B). Intraoperative cholangiography could not be employed since the cardiopulmonary status could not tolerate additional operating time. Although the underlying pathology was not clear, we speculated the possibility of bile transudation through the biliary tract. We performed cholecystectomy followed by irrigation and drainage of the abdominal cavity. Postoperatively, she was treated empirically with broad-spectrum antibiotics. On gross examination, the resected gallbladder was $9.8 \mathrm{~cm}$ long without gallstone. There were focal thinning parts in the corpus of the resected gallbladder, and bile leakage from where was not identified macroscopically (Figure 3A). Histopathological findings showed an infiltration of the inflammatory cells into the wall of gallbladder (Figure 3B), but neither necrosis nor perforation were detected. Postoperative course was uneventful. She underwent gastroduodenoscopy which could not identify any suspicious finding of perforation in the gastroduodenal tract but revealed a large parapapillary diverticulum (Figure 4).

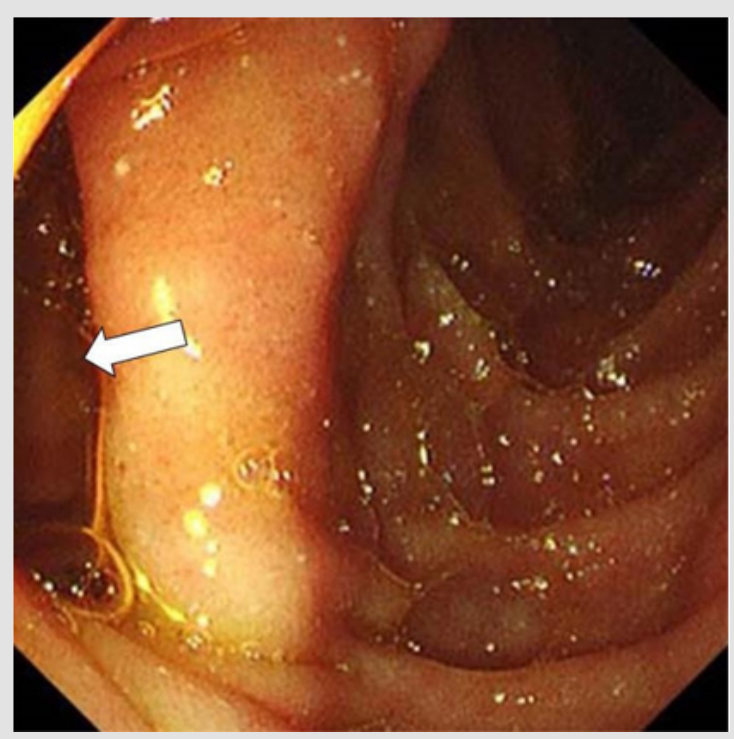

Figure 4: Postoperative gastroduodenoscopy revealed alarge parapapillary diverticulum (arrow).

Postoperative Magnetic Resonance Cholangiopancreatography (MRCP) did not show anomalous arrangement of pancreaticobiliary ductal system and choledocholithiasis (Figure 5). We recommended endoscopic retrograde cholangiopancreatography and endoscopic retrograde biliary drainage to the patient, but she refused any invasive examinations and treatments. Oral intake was initiated on the 10 days after surgery and laboratory findings associated with inflammation and obstruction of the biliary tract were not increased even after returning oral feeding. She was eventually discharged on the $72^{\text {nd }}$ postoperative day.

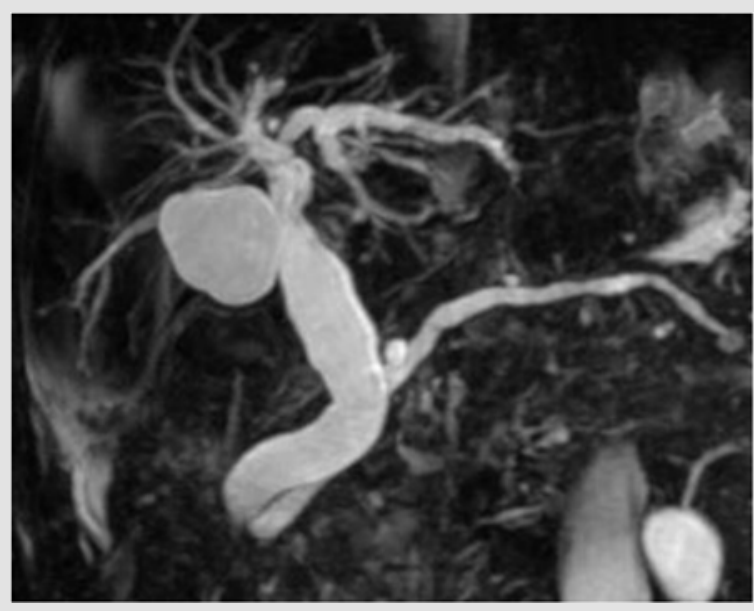

Figure 5: Postoperative MRCP did not show anomalous arrangement of pancreaticobiliary ductal system and choledocholithiasis.

\section{Discussion}

Biliary peritonitis is a relatively rare clinical entity and is usually caused by the perforation of the biliary system or upper gastrointestinal tract $[1,2]$. On the other hand, the cases of biliary peritonitis without any macroscopic perforation of the biliary tract were extremely rare and have been previously described in acute cholecystitis, trauma, and choledocholithiasis [11,12]. There also have been reported the cases of biliary peritonitis occurring without biliary pathology of any kind $[11,12]$. The pathogenesis of this rare clinical phenomenon has not been established, but several possible mechanisms have been advocated, such as:

(i) bile leak through the Rokitansky-Achoff sinus invaginated into the thinning part of the gallbladder wall,

(ii) ischemia of the bile tract.

which is caused by arteriosclerosis, arterial thrombosis, and compromised venous drainage caused by acute increase in the intraductal pressure;

(iii) regurgitation of pancreatic juice into the bile duct due to anomalous arrangement of pancreaticobiliary ductal system which results in autodigestion and chemical inflammation of the biliary tract mucosa;

(iv) intraluminal infection which contributes to wall weakness and lower resistance to the increase of intraductal pressure;

(v) other reasons such as biliary tract malignancies, corticosteroid treatments, and diabetes mellitus $[4,5,10]$. In the present case, the histopathological evaluation did not reveal either RAS or ischemic changes in the resected gallbladder.

The postoperative MRCP did not show anomalous arrangement of pancreaticobiliary ductal system. Although we could not determine certain etiology of the bile leak through the biliary tract, 
the putative cause seems to be associated with the mechanical manipulation of the colon during colonoscopy and steroid-induced connective tissue fragility of the biliary tract wall. No previous report of the biliary peritonitis without any perforation of biliary tract occurring after colonoscopy has been reported. Repetitive traction on the adhesions between the gallbladder, common bile duct, colon, and omentum during the colonoscopy could be leading to a torsion of the biliary tract since a similar mechanism has been proposed for splenic rupture after colonoscopy in the presence of adhesions between the colon and spleen [13]. However, we did not find any adhesions and injuries of the biliary tract around the gallbladder and hepatic hilum. Although its pathophysiology is uncertain, acute cholecystitis has been reported as an extremely rare complication of colonoscopy [14]. The theorized pathogenesis is the transient relative dehydration following bowel preparation which causes the bile to become more lithogenic, and bile stasis leading to gallbladder distention [14]. Other proposed mechanisms are mechanical manipulation, and the associated bacterial translocation of the colon may also play a role in the secondary infection of the gallbladder with enteric pathogens [14].

In the present case, we speculated that bacterial translocation via mechanically damaged colon mucosa during colonoscopy might cause an infection and subsequent inflammation of the biliary tract with enteric pathogens, which weakened the biliary duct wall and decreased its resistance to intraductal pressure. The intraductal pressure of the biliary tract might be elevated due to a large parapapillary diverticulum since preoperative CT revealed dilated common bile duct. Additionally, the fragility of the biliary tract wall due to a long-term administration of corticosteroid might resulted in the transudation of bile juice. Idiopathic biliary peritonitis is difficult to be diagnosed before the treatment such as surgical operation. The delay of diagnosis is associated with high morbidity and mortality in surgical operation [6-12]. Ultrasonography and CT were informative modalities for detecting the abnormalities in the biliary tract and abnormal fluid collection in the perihepatic space $[9,10]$. Furthermore, abdominal paracentesis with appropriate ascitic fluid analysis is a simple and helpful adjunct for the diagnosis of biliary peritonitis [10]. In the present case, we highly suspected the colonic perforation due to colonoscopy since abnormal ascites was formed mainly in the left paracolic gutter, and the laparoscopic examination could provide the correct diagnosis of biliary peritonitis. Surgical intervention is the mainstay of treatment for the biliary peritonitis. It is important to drain abdominal contaminations caused by infected bilious peritoneal fluid $[9,10,15]$.

In most cases, cholecystectomy and drainage of the abdominal cavity have been performed for the biliary peritonitis with gallbladder perforation [7,10]. Cholecystectomy might not be essential in the case without detectable perforation of the biliary tract, but we considered that cholecystectomy should be performed since the possibility of bile transudation through the gallbladder could not be excluded.

\section{Conclusion}

Biliary peritonitis remains as a rare cause of acute abdomen. Immediate diagnosis and surgical intervention are the mainstays of treatment to improve the morbidity and mortality in the cases with biliary peritonitis. Therefore, idiopathic biliary peritonitis should be taken into consideration in differential diagnosis for the acute abdomen.

\section{References}

1. Shukla RM, Roy D, Mukherjee PP, Saha K, Mukhopadhyay B, et al. (2011) Spontaneous gall bladder perforation: A rare condition in the differential diagnosis of acute abdomen in children. J Pediatr Surg 46: 241-243.

2. Namikawa T, Kobayashi M, Okabayashi T, Okamoto K, Akimori T, et al. (2007) Clinicopathological analysis of idiopathic perforation of the gallbladder. Surg Today 37: 633-637.

3. Kent SJ, Menzies Gow N (1974) Biliary peritonitis without perforation of the gallbladder in acute cholecystitis. Br J Surg 61: 960-962.

4. Ohashi K, Sasaki A, Ohta K, Matsuo Y (2011) A Case of Billiary Peritonitis due to Transudation through the Gallbladder. Jpn J Gastroenterol Surg 44(2): 152-158.

5. Aosasa S, Morita D, Oka A, Chochi K (2012) A case of biliary peritonitis due to bile leakage through the gallbladder. J Jpn Surg Assoc 73(7): 1817-1821.

6. Talwar N, Andley M, Ravi B, Kumar A (2006) Spontaneous biliary tract perforations: An unusual cause of peritonitis in pregnancy. Report of two cases and review of literature. World J Emerg Surg 1(1): 21.

7. Shukla RM, Roy D, Mukherjee PP, Saha K, Mukhopadhyay B, et al. (2011) Spontaneous gall bladder perforation: A rare condition in the differential diagnosis of acute abdomen in children. J Pediatr Surg 46: 241-243.

8. Gundara JS, Jancewicz S (2011) Spontaneous biliary peritonitis, or delayed bile leak? Int J Surg Case Rep 2(6): 166-167.

9. Kang SB, Han HS, Min SK, Lee HK (2004) Nontraumatic perforation of the bile duct in adults. Arch Surg 139: 1083-1087.

10. Hamura R, Haruki K, Tsutsumi J, Takayama S, Shiba H, et al. (2016) Spontaneous biliary peritonitis with common bile duct stones: Report of a case. Surgical Case Reports 2: 103.

11. Cope Z (1925) Extravasation of bile. Br J Surg 13(49): 120-129.

12. Ellis H, Cronin K (1961) Bile peritonitis. Br J Surg 48: 166-171.

13. Sarhan M, Ramcharan A, Ponnapalli S (2009) Splenic injury after elective colonoscopy. JSLS 13(4): 616-619.

14. Cassidy Campbell, Edward A Cooper, Philip Kennedy K (2020) Emphysematous cholecystitis following routine colonoscopy. JSCR 4: $1-2$

15. So kmen S, Coker A, Unek T (2001) Spontaneous biliary peritonitis in acalculous cholecystitis: Fact or misdiagnosis? Hepatogastroenterology 48: $1001-1004$ 
ISSN: 2574-1241

DOI: 10.26717/BJSTR.2021.33.005423

Yoshihito Souma. Biomed J Sci \& Tech Res

(C) (P) This work is licensed under Creative

Submission Link: https://biomedres.us/submit-manuscript.php

$\begin{array}{ll}\text { BIOMEDICAL } & \text { Assets of Publishing with us } \\ \text { RESEARCHES } & \text { - Global archiving of articles } \\ \text { - Immediate, unrestricted online access }\end{array}$

